\title{
ATLAS measurement of Electroweak Vector Boson production
}

\author{
C. Vittori ${ }^{\mathrm{a}}$ \\ on behalf of the ATLAS Collaboration \\ ${ }^{a}$ INFN and University of Bologna, via Irnerio 46 Bologna, Italy
}

\begin{abstract}
The measurements of the Drell-Yan production of $W$ and $Z / \gamma^{*}$ bosons at the LHC provide a benchmark of our understanding of the perturbative QCD and probe the proton structure in a unique way. The ATLAS collaboration has performed new high precision measurements of the double differential cross-sections as a function of the dilepton mass and rapidity. The measurements are compared to state of calculations at NNLO in QCD and constrain the photon content of the proton. The angular distributions of the Drell-Yan lepton pairs around the Z-boson mass peak probe the underlying QCD dynamics of the Z-boson production mechanisms. The complete set of angular coefficients describing these distributions is presented and compared to theoretical predictions highlighting different approaches of the QCD and EW modelling. First precise inclusive measurements of $\mathrm{W}$ and $\mathrm{Z}$ production at $13 \mathrm{TeV}$ are presented. $W / Z$ and $W^{+} / W^{-}$ratios profit from a cancellation of experimental uncertainties.
\end{abstract}

Keywords: High mass Drell Yan, Angular coefficients, $\mathrm{Z}$ and $\mathrm{W}$ cross sections at $13 \mathrm{TeV}, W^{+} / W^{-}$and $W / Z$ ratios, $Z+$ jets cross section

\section{Introduction}

The measurements of electroweak vector-boson production at hadron colliders provide a benchmark for the understanding of quantum chromodynamics (QCD) and electroweak (EW) processes. Predictions are available up to next-to-next-to-leading-order (NNLO) accuracy in QCD and include EW corrections at next-to-leadingorder (NLO) accuracy. The cross-section predictions depend on Parton Distibutions Functions (PDFs) and are thus sensitive to the underlying dynamics of strongly interacting particles. Therefore, the measurements of $W^{ \pm}$ and Z-bosons production offer the opportunity to test the model of partonic interactions at LHC.

All measurements in this paper have been performed by the ATLAS experiment [1]. In Section 2, the most recent results on High Mass Drell-Yan production cross section at $8 \mathrm{TeV}$ are presented. In Section 3, the angular coefficients measurements for $\mathrm{Z}$ events at $8 \mathrm{TeV}$ are introduced. In Section 4 , the $W^{ \pm}$and Z-bosons production cross sections values and ratios at $13 \mathrm{TeV}$ are presented; in particular in Section 5, the results on the measurements of the $\mathrm{Z}$ cross section production in association with jets at $13 \mathrm{TeV}$ are shown.

\section{High-Mass Drell-Yan at $8 \mathrm{TeV}$}

Although on-shell $p p \rightarrow Z+X$ (with $Z \rightarrow l^{+} l^{-}$) process measurement provides the greatest experimental precision, they are restricted in the kinematic range of partonic momentum fractions $x$ and four-momentum transfer $Q=m_{l l}$, the invariant mass of the dilepton pair. On the other hand, the off-shell measurements $p p \rightarrow \gamma^{*}+X$ provide complementary constraints in a wider range of $x$ and $Q$. The measurements have different sensitivity to the up- and down-quark distributions and at large $m_{l l}$ they provide constraints on the large- $x$ anti-quark PDFs. Moreover, the off-shell measurements are sensitive to the photon PDF, trough the photon-induced (PI) process $\gamma \gamma \rightarrow l^{+} l^{-}$.

The inclusive single-differential cross-section $d \sigma / d m_{l l}$ and two double-differential cross-sections 
$d^{2} \sigma / d m_{l l} d\left|y_{l l}\right|$ and $d^{2} \sigma / d m_{l l} d\left|\Delta \eta_{l l}\right|$ have been measured 92 with data collected at $\sqrt{s}=8 \mathrm{TeV}$ in both electron and muon channels with an integrated luminosity of $20.3 \mathrm{fb}^{-1}$ [2]. Data cover the kinematic region of $116 \leq m_{l l} \leq 1500 \mathrm{GeV}$ and access a partonic momentum fraction from $10^{-3}$ up to $x \sim 1$.

Electrons and muon selections have been optimised separately; the common fiducial phase space at Bornlevel includes two opposite-charged leptons with $p_{T}^{l}>30(40) \mathrm{GeV}$ for the leading (sub-leading) lepton in the mass window $116 \leq m_{l l} \leq 1500 \mathrm{GeV}$.

Signal and background samples (Top 9\% and diboson $\sim 2 \%$ ) have been simulated with different Monte Carlo generators, with the exception of the multi-jet background, treated with data-driven methods $(\sim 4 \%$ and $\sim 2 \%$ in electron and muon channel respectively).

The measured Born-level fiducial cross-sections for the electron and muon channels are in good agreement with one another and achieve a precision of $1 \%$ at low $m_{l l}$, where they are dominated by the experimental systematic uncertainties [2]. The differential cross section as a function of $m_{l l}$ and $\left|y_{l l}\right|$ shows a marked narrowing of the rapidity plateau width as $m_{l l}$ increases, while the cross section as a function of $m_{l l}$ and $\left|\Delta \eta_{l l}\right|$ is largest where the lepton separation is close to zero and decreases where $\left|\Delta \eta_{l l}\right|$ increases. The cross section measurements at Born-level have been compared to NNLO pQCD calculations, obtained using various PDFs. The calculations include NLO EW corrections and the non-resonant PI-process, estimated at $\mathrm{LO}$ using the photon PDF from the NNPDF2.3qed PDF set [2].

The theoretical predictions using the MMHT14 NNLO 100 PDF set are compared to the double-differential 101 fiducial cross-sections. The uncertainties assigned to 102 the calculations involve the PDFs, the $\alpha_{s}$ variation, 103 the renormalization and factorization scales and the 104 PI-process uncertainties. Comparing the ratio of the 105 default calculation with the calculation neglecting the ${ }_{106}$ PI contribution, the PI contribution reaches the $15 \%$ in 107 certain regions of phase space.

In order to quantitatively test the compatibility between 109 data and theory, a $\chi^{2}$ minimisation procedure is used, 110 taking into account all correlated and uncorrelated 111 experimental, luminosity and theoretical uncertainties. ${ }_{112}$ The $\chi^{2}$ minimisation is performed separately for all ${ }_{113}$ PDF sets and indicates a general compatibility between 114 data and theory.

The impact of data on PDF sets can be inferred using ${ }_{116}$ Bayesian reweighting: one hundred NNPDF replicas 117 have been created and have been used with data in the ${ }_{118}$ $\chi^{2}$ minimisation; then a Bayesian weight based on the ${ }_{119}$ resulting $\chi^{2}$ has been assigned to each replica. Replicas ${ }_{120}$ not describing the data get a smaller weight and a new PDF is derived including the weights in the calculation. As can be inferred from figure 1, the PDF built with the reweighting procedure based on data shows a reduced uncertainty band in the photon PDF, which confirms the strong sensitivity of data to the photon PDF.

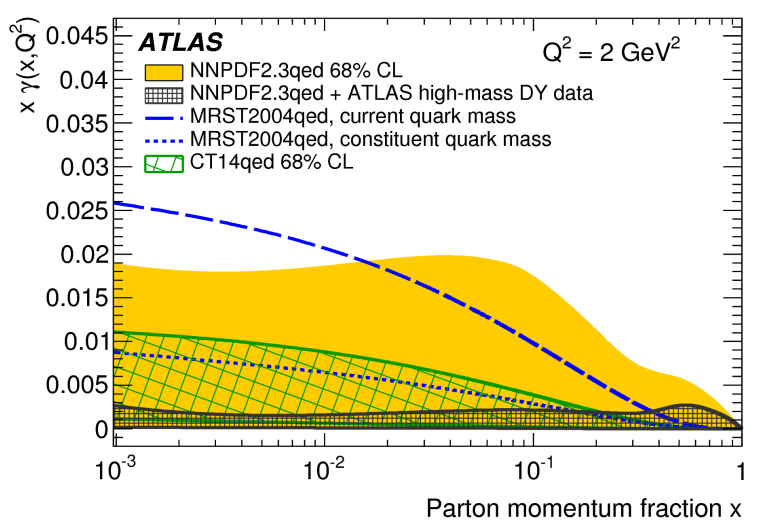

Figure 1: The $68 \%$ confidence interval of the NNPDF2.3qed NNLO photon PDF as a function of momentum fraction $x$ at the input scale of $Q^{2}=2 \mathrm{GeV}^{2}$, before (yellow solid area) and after (black shaded area) the inclusion of the double-differential cross section measurements [2].

\section{Angular Coefficients in $\mathrm{Z}$ events at $8 \mathrm{TeV}$}

The angular distributions of charge lepton pairs produced in $p p$ collision via the Drell-Yan neutral current process provide a portal to precise measurements of the production dynamics through the spin correlation effects between the initial and final states. In the Z-boson rest frame, a plane spanned by the directions of the incoming protons can be defined, e.g. the "CollinsSoper" (CS) rest frame [3], where the lepton polar and azimuthal angular variables, denoted by $\cos \theta$ and $\phi$, are defined. The spin correlations are described by a set of nine helicity density matrix elements, which can be evaluated in the perturbative QCD. The fivedimensional differential cross-section which describes the kinematics of the two Born-level leptons from the Z-boson decay, can be decomposed as a sum of nine harmonic polynomials [3] which are functions of $\cos \theta$ and $\phi$. Factorising the unpolarised cross-section, the fivedimensional cross-section is presented as an expansion into nine harmonic polynomials $P_{i}(\cos \theta, \phi)$ and dimensionless angular coefficients $A_{0-7}\left(p_{T}^{Z}, y^{Z}, m^{Z}\right)$ [3]. Given that the dependence on $p_{T}^{Z}, y^{Z}$ and $m^{Z}$ is entirely contained in the $A_{i}$ coefficients, precise measurements al- 
low a direct access to the dynamics of the event. Templates of the nine polynomials folded to the detector level are fitted to the data to extract the angular coefficients in the full phase space of the $\mathrm{Z}$ boson. At LO QCD only the annihilation diagram $q \bar{q} \rightarrow Z$ is present and only $A_{4}$ is non-zero; at NLO QCD $A_{0-3}$ also become non-zero. The Lam-Tung relation predicts $A_{0}-A_{2}=0$ due to the spin- 1 of the gluon in the $q g \rightarrow Z q$ and $q \bar{q} \rightarrow Z g$ diagrams; it is expected to hold up to $O\left(\alpha_{s}\right)$ and to be violated at higher orders. Arising from gluon loops, the coefficients $A_{5,6,7}$ are predicted to become non-zero only at NNLO QCD.

The analysis has been performed in three different channels, requiring two central muons, two central electrons and one central and one forward electrons through specific selection criteria [3]. Due to the requirement of an invariant mass within a narrow window around the Z-boson peak $\left(80<m_{l l}<100 \mathrm{GeV}\right)$, the background contribution is estimated to be less than $0.5 \%$. Most of the phase space measurements are limited only by the statistical uncertainties in the data, which range from 0.002 at $p_{T}^{Z}=5 \mathrm{GeV}$ to 0.008 at $p_{T}^{Z}=150 \mathrm{GeV}$. The experimental systematic uncertainties are much smaller and theoretical uncertainties, dominated by the PDFs, are below 0.004 .

When compared to theoretical calculations and to $\mathrm{MC}$ predictions, the measured coefficients are precise enough to probe QCD corrections beyond the accuracy of calculations [3]. $A_{0,3,4}$ are well described by fixed order calculations, while evidence at the $3 \sigma$ level is found for non-zero $A_{5,6,7}$ coefficients, consistent with expectations from DYNNLO [3]. The experimental results confirm that the Lam-Tung relation $\left(A_{0}-A_{2}=0\right)$ does not hold at $O\left(\alpha_{s}\right)$ : in figure 2, significant deviations from ${ }^{181}$ zero (almost a factor two larger than predictions) are observed for $p_{T}^{Z}>50 \mathrm{GeV}$, indicating that higher-order 182 QCD corrections are required to describe the experi- 183 mental results.

The measurements also provide discrimination between 185 various event generators, in particular in term of the re- 186 lated implementation of different parton-shower mod- ${ }_{187}$ els [3]. The measurements of the $A_{i}$ coefficients are an ${ }_{188}$ important ingredient to the next steps in precision mea- 189 surements of electroweak parameters at the LHC (e.g. 190 the weak mixing angle $\sin _{W}^{2}$ and the $\mathrm{W}$-mass).

\section{The measurements of the $W^{ \pm}$and Z-bosons pro- duction cross sections at $13 \mathrm{TeV}$}

Measurements of electroweak vector-boson produc- 197 tion at LHC provide a benchmark for perturbative QCD 198

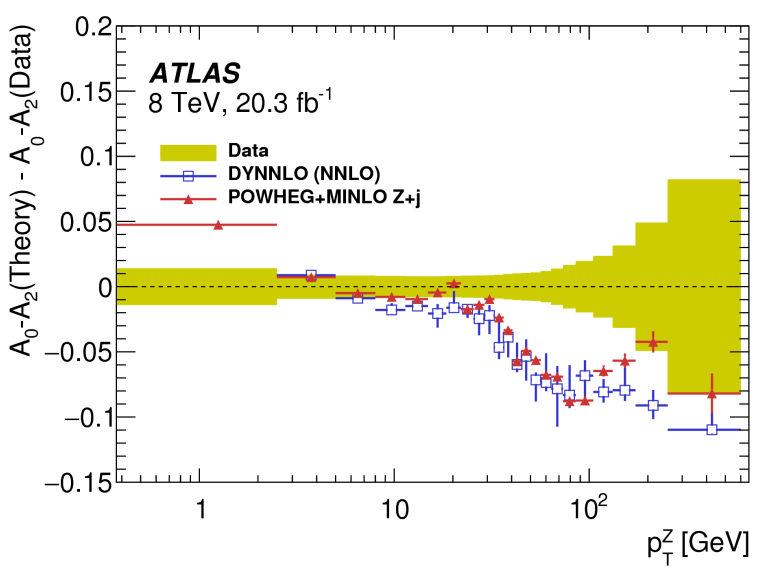

Figure 2: Distributions of the differences between calculations and data in the measurement of $A_{0}-A_{2}$ as a function of $p_{T}^{Z}$. The results are compared to the DYNNLO and POWHEG MINLO. The shaded band represent the total uncertainties in the measurements [3].

and are extremely useful to constrain PDFs, being sensitive to the dynamics of strongly interacting particles. The $\mathrm{Z}$ cross section is a powerful tool for detector alignment, calibration and luminosity monitoring, through the Z-counting technique.

Inclusive $\mathrm{W}$ and $\mathrm{Z}$ cross sections have been measured in the electron and muon channels with data corresponding to an integrated luminosity of $81 \mathrm{pb}^{-1}$, collected at $\sqrt{s}=13 \mathrm{TeV}$ by the ATLAS detector [4]. The total production cross sections times the branching ratio for decays into a single-lepton flavour can be expressed as:

$$
\sigma=\frac{N}{\mathcal{L} \cdot A \cdot C},
$$

where $\mathrm{N}$ is the number of data observed subtracted by the background events and $\mathcal{L}$ is the total integrated luminosity. The acceptance factor $A$ is expressed as the fraction of decays satisfying the fiducial W/Z acceptance (geometric and kinematic requirements) at generator level. The correction factor $C$ represents the ratio of the total number of generated events which pass the final selection requirements after reconstruction to the total number of generated events within the fiducial acceptance. This factor, defined before the decay leptons emit photons via final-state radiation (Born-level before FSR), includes the efficiencies for triggering on, reconstructing, and identifying the $\mathrm{W}$ and Z-boson decay products within the acceptance, and also accounts for the slight difference between the fiducial and reconstructed phase space.

For the $\mathrm{W}$ measurements, the fiducial phase space is de- 
fined by the lepton transverse momentum $p_{T}^{l}>25 \mathrm{GeV}$ and pseudo-rapidity $\left|\eta_{l}\right|<2.5$, neutrino transverse momentum $p_{T}^{v}>25 \mathrm{GeV}$ and $\mathrm{W}$ transverse mass $m_{T}^{W}>50$ $\mathrm{GeV}$; similarly the $\mathrm{Z}$ boson production is measured in the same fiducial phase space defined by $p_{T}^{l}>25 \mathrm{GeV}$, $\left|\eta_{l}\right|<2.5$ and $66<m_{l l}<116 \mathrm{GeV}$.

Predictions are available up to NNLO QCD and include EW corrections up to NLO accuracy. The theoretical systematic uncertainties are dominated by the knowledge of the PDFs; renormalisation and factorisation scales and the strong coupling constant follow.

Contributions from the electroweak (single boson and diboson) and top quark (single-top and top quark pairs) background components are estimated from Monte Carlo samples. The $\mathrm{W} \rightarrow \tau v$ and $\mathrm{Z} \rightarrow \tau \tau$ processes with the subsequent leptonic decay of the $\tau$, are also considered as background sources. The multijet contribution in the $\mathrm{W}$ analysis is estimated with a data-driven approach, performing maximum-likelihood fits on the data with template distributions [4] of the following variables: $m_{T}, E_{T}^{m i s s}, p_{T}^{l}$ and $\Delta \phi$ between the lepton and the transverse missing momentum. The estimated multijet background fractions of the total number of observed events are $8 \%$ and $10 \%$ in the electron and 3.5\% and $4 \%$ in the muon $W^{+}$and $W^{-}$channels. The multijet background in the Z-boson analysis is estimated from simulation to be $<0.1 \%$ and is therefore neglected.

The ratio of the electron and muon channel measurements in the $W^{ \pm}\left(R_{ \pm}=\sigma_{W^{ \pm} \rightarrow e v} / \sigma_{W^{ \pm} \rightarrow \mu v}\right)$ and $Z(R=$ $\left.\sigma_{Z \rightarrow e^{+} e^{-}} / \sigma_{Z \rightarrow \mu^{+} \mu^{-}}\right)$channels have been evaluated taking into account correlations. Since the results agree well with the Standard Model expectations of lepton universality, a simultaneous combination of the $W^{+}, W^{-}$and Z-boson cross sections is performed [4]. The combined ${ }_{250}$ measurements are compared in figure 3 with predictions, which have been calculated using different PDF ${ }_{251}$ sets. The measured cross sections have a global lumi- 252 nosity uncertainty of $2.1 \%$, while their remaining exper- ${ }_{253}$ imental uncertainties in the $W^{ \pm}$and Z-boson channels ${ }_{254}$ are found to be just under $3 \%$ and $1 \%$, respectively. $\quad 255$

The ratios of the measured cross sections benefit from 257 the cancellation of some experimental uncertainties (lu- 258 minosity) and constrain the PDF fits. In particular, the ${ }_{259}$ ratio $W^{+} / W^{-}$is mostly sensitive to the difference of 260 $u$ and $d$ valence-quark distributions at low Bjorken-x, 261 while the ratio $W^{ \pm} / Z$ constrains the $s$-quark distribution. 262 The cross-section $W^{+} / W^{-}$ratio, measured with an un- 263 certainty of $0.8 \%$, is able to discriminate amongst the 264 various PDF predictions, as presented in figure 4 . These 265 results form a basis for further tests of perturbative QCD 266 and exploration of the partonic content of the proton. $\quad{ }_{267}$

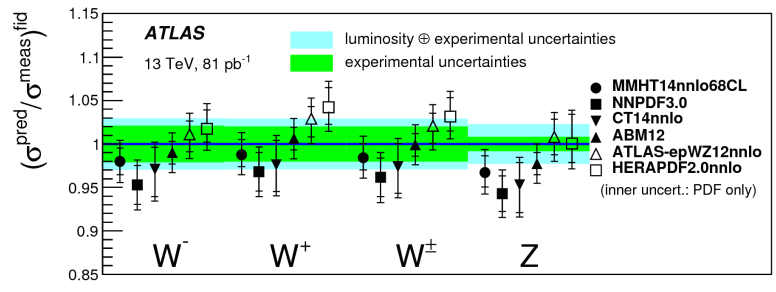

Figure 3: Ratio of the predicted to measured cross sections for the combined electron and muon channels using various PDFs. The inner (outer) band corresponds to the experimental uncertainty without (with) the luminosity uncertainty [4].

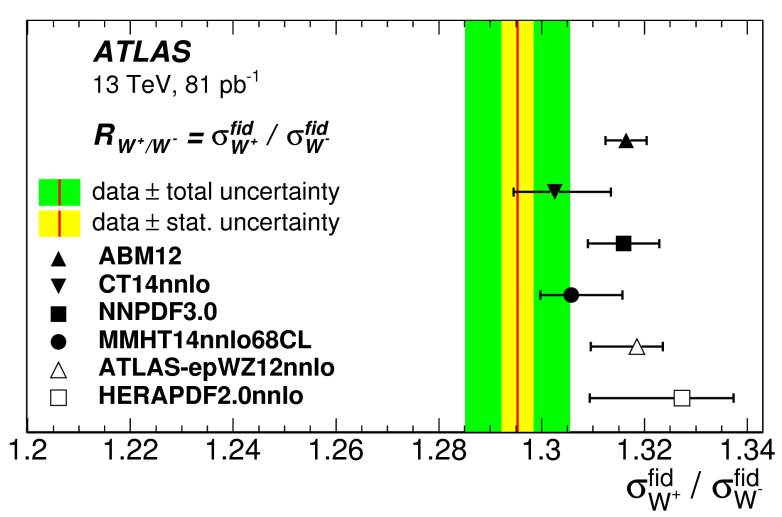

Figure 4: Ratio of $W^{+} / W^{-}$combined production cross sections compared to predictions based on different PDF sets. The inner (yellow) shaded band corresponds to the statistical uncertainty while the outer (green) band shows statistical and systematic uncertainties added in quadrature [4].

\section{5. $\mathrm{Z}$ production in association with jets at $13 \mathrm{TeV}$}

The $W / Z$ events produced in association with jets provide an important test of perturbative QCD and constitute a non-negligible background for studies of the Higgs boson and searches of new phenomena. The predictions from the most recent generators combine NLO multi-leg matrix elements with a parton shower.

The $\mathrm{Z}+$ jets production cross section has been measured with data corresponding to an integrated luminosity of $81 \mathrm{pb}^{-1}$, collected at $\sqrt{s}=13 \mathrm{TeV}$ by the ATLAS detector [5]. The $\mathrm{Z}$ boson is identified using its decays to electron and muon pairs with the same strategy employed in the inclusive analysis (Section 4). Hadronic jets are reconstructed through the anti-kt algorithm with radius parameter $\mathrm{R}=0.4$, using topological cluster in the calorimeter as input. Jets are calibrated using an $E^{T}$. and $\eta$-dependent simulation-based calibration scheme and are selected with transverse momentum $p_{T}>30$ 
$\mathrm{GeV}$ and rapidity $|y|<2.5$; jets closer than 0.4 in $\Delta R=$ $\sqrt{\Delta \phi^{2}+\Delta \eta^{2}}$ to a selected lepton are discarded.

Top quark pair production is the dominant background for higher jet multiplicities: it contributes around 1\% in the $\mathrm{Z} \rightarrow l^{+} l^{-}+\geq 1$ jet samples and rises from $5 \%$ to $10 \%$ going from $\mathrm{Z} \rightarrow l^{+} l^{-}+\geq 2$ jets $\mathrm{Z} \rightarrow l^{+} l^{-}+\geq 4$ jets event selections. Minor background contributions arise from diboson and single-boson decaying to leptonic final states. The multijet background is negligible in the $\mathrm{Z} \rightarrow \mu^{+} \mu^{-}$channel and at most at the percent level in the $\mathrm{Z} \rightarrow e^{+} e^{-}$one.

The agreement between data and predictions has been evaluated from the comparison of several kinematic distributions, like the dilepton mass, the inclusive jet multiplicity and the transverse momentum and rapidity of the leading jet, to both MadGraph and Sherpa Monte Carlo generators. Both MadGraph and Sherpa simulation predictions show a reasonable agreement to the data.

The cross sections for various jet multiplicities and their ratios for those with larger jet multiplicities have been measured in both electron and muon channels and compared with predictions [5].

In order to improve the precision of the measurement, the result in the $\mathrm{Z} \rightarrow \mu^{+} \mu^{-}$and $\mathrm{Z} \rightarrow e^{+} e^{-}$channel have been combined, taking into account the correlated systematic uncertainties. The combination is performed for ${ }_{315}$ the inclusive $\mathrm{Z}$ boson cross section and for the various 316 jet multiplicities at the Born-level after the evaluation of ${ }^{317}$ the Born-to-dresses level cross section correction. $\quad{ }_{318}$ The combined cross section is compared with the pre- ${ }_{320}$ dictions in figure 5, where a good agreement between ${ }^{32}$ data and predictions can be observed within the uncer- ${ }^{322}$ tainties.

\section{Conclusions}

The electroweak vector bosons measurements are extremely important to improve the understanding of QCD processes. Drell-Yan double-differential cross sections have been measured by ATLAS at $8 \mathrm{TeV}$ with a precision better than $1 \%$ at low mass, allowing to set constraints on proton PDFs and to be sensitive to photon PDF. Spin correlations between initial and final leptonic states have been evaluated. W, Z and Z+jets cross sections and their ratios have been measured at $13 \mathrm{TeV}$, reaching a precision comparable to that from the PDF uncertainties.

\section{References}

[1] ATLAS Collaboration, 2008 JINST 3 S08003.

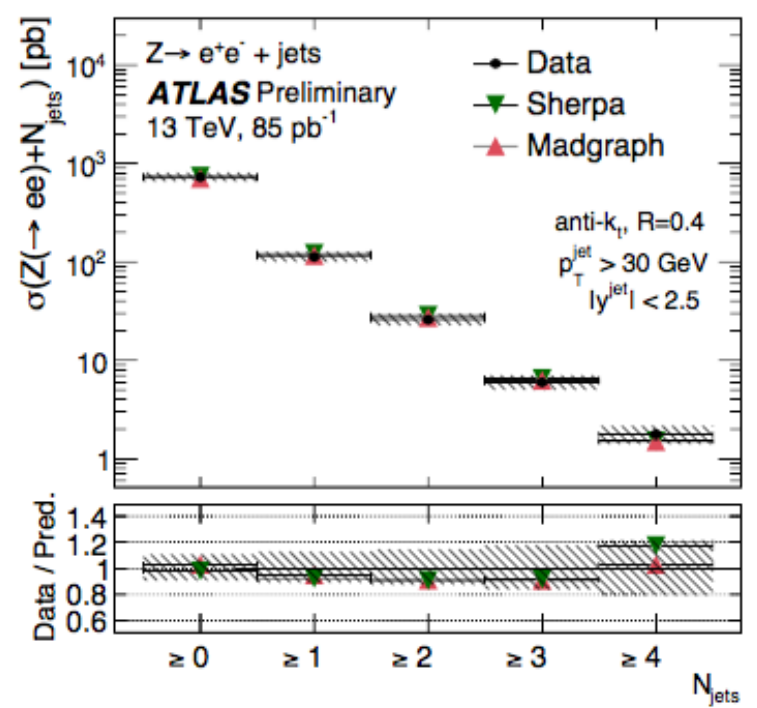

Figure 5: Combined $\mathrm{Z} \rightarrow l^{+} l^{-}+\geq \mathrm{N}$ jets fiducial cross sections. Results are reported at the Born-level and compared to predictions from Sherpa and MadGraph [5]. On the bottom, the ratio between data and predictions is shown.

[2] ATLAS Collaboration, "Double Differential High-mass DrellYan production at $\sqrt{s}=8 \mathrm{TeV}$ ", arXiv:1606.01736 - JHEP 08 (2016) 009.

[3] ATLAS Collaboration, "Angular coefficients in Z-boson event at $\sqrt{s}=8 \mathrm{TeV}$ ", arXiv:1606.00689 - submitted to JHEP.

[4] ATLAS Collaboration, "Measurement of $W^{ \pm}$and Z-boson production cross sections in pp collisions at $\sqrt{s}=13 \mathrm{TeV}$ with the ATLAS detector", arXiv:1603.09222 - Phys. Lett. B 759, 601 (2016).

[5] ATLAS Collaboration, "Measurement of the Production Cross Sections of a Z Boson in Association with Jets in pp collisions at $\sqrt{s}=13 \mathrm{TeV}$ with the ATLAS Detector", ATLAS-CONF2015-041. 